\title{
Very Low Potential Electrodeposition of Sm-Co Nanostructures in Aqueous Medium Using Hard Templates
}

\author{
Elisa Herrera, ${ }^{1}$ Julieta Riva, ${ }^{2,3}$ Gabriela Pozo-López, ${ }^{1,2,3}$ Adriana Condó, \\ Silvia E. Urreta, ${ }^{2}{ }^{2}$ and Luis M. Fabietti ${ }^{1,2,3, z}$ \\ ${ }^{1}$ Instituto de Física Enrique Gaviola - CONICET, Córdoba, Argentina \\ ${ }^{2}$ Universidad Nacional de Córdoba Facultad de Matemática, Astronomía, Física y Computación, Ciudad \\ Universitaria, 5000 Córdoba, Argentina \\ ${ }^{3}$ Consejo Nacional de Investigaciones Científicas y Técnicas (CONICET), Argentina \\ ${ }^{4}$ Centro Atómico Bariloche, Comisión Nacional de Energía Atómica - Instituto Balseiro, Universidad Nacional de \\ Cuyo, 8400 San Carlos de Bariloche, Argentina
}

\begin{abstract}
Arrays of SmCo nanowires (NW) and nanotubes (NT) with a diameter of $200 \mathrm{~nm}$ and different lengths are synthesized by electrodeposition into the nanopores of an alumina membrane. The potential applied during the synthesis largely determines the nanostructure morphology, its crystallinity and composition. Potentials investigated are between $-0.8 \mathrm{~V}$ and $-3.0 \mathrm{~V}$; in the potential range between $-0.8 \mathrm{~V}$ and $1.0 \mathrm{~V}$, long and perfectly ordered nanowires are obtained with a composition close to that of the equilibrium $\mathrm{Sm}_{2} \mathrm{Co}_{17}$ phase in the binary alloy. For higher potentials, above $-1 \mathrm{~V}$, samples are nanotubes, $195 \mathrm{~nm}$ in external diameter and wall thickness of $30 \mathrm{~nm}$ with an equiatomical composition. Magnetic characterization reveals that all the nanostructures are soft ferromagnetic, with coercivity values below $60 \mathrm{mT}$. From the angular dependence of coercivity and the relative remanence it may concluded that in both, nanowires and nanotubes the magnetization reversal mechanism undergoes a transition from one at smaller angles, involving localized nucleation by curling, and further expansion of vortex-like domain walls. At higher angles, where the applied field is almost perpendicular to the NW/NT long axis, the mechanism changes to one involving nucleation by localized coherent rotation and further expansion of transverse Bloch-like walls.
\end{abstract}

(C) 2019 The Electrochemical Society. [DOI: 10.1149/2.1311910jes]

Manuscript submitted March 26, 2019; revised manuscript received May 16, 2019. Published June 21, 2019.

There has been an explosive growth of nanoscience and nanotechnology in the last few years, primarily because of the availability of new strategies for the synthesis of nanomaterials and new tools for their characterization and manipulation. Nanostructures and nanomaterials dominate the research scene in almost all areas of natural sciences and engineering worldwide due to their countless applications in different areas of knowledge such as medicine, pharmacology, photonic, spintronic and many others. ${ }^{1-3}$ In this sense, it is possible to see in the literature new ways to synthesize and characterize nanostructures with different morphologies and dimensionalities, and fundamentally new materials designed for specific applications. ${ }^{4,5}$

Magnetic nanowires have been widely investigated in connection with their potential applications in magnetic and electronic nanodevices. ${ }^{6}$ Magnetically hard ferromagnetic alloys containing rare earths have been specially studied in nanowire morphology looking for an improvement in their hysteresis properties in connection with a large shape anisotropy contribution to coercivity. ${ }^{7-10}$ Samarium alloys, ferromagnetically coupled with $\mathrm{Fe}, \mathrm{Co}$ and $\mathrm{Ni}$, are among some of the strongest known permanent magnets. ${ }^{11}$ Phases such as $\mathrm{SmCo}_{5}$ and $\mathrm{Sm}_{2} \mathrm{Co}_{17}$ lead to materials with quite high magnetic moment, anisotropy and coercivity ${ }^{12}$ which is controlled by the domain wall pinning by grain boundaries. One of the advantages of SmCo alloys is that the Curie temperature is on the order of $700^{\circ} \mathrm{C}$, which is more than twice compared to the Nd-based alloys. However, these alloys have the disadvantage that a nanometric grain size is required to generate optimal magnetic properties, and this implies the use of drastic and complex processing conditions such as high potential application or the use of non aqueous solvents. ${ }^{13-16}$ The primary way to prepare rare earth-transition metal alloy thin films has been the vacuum deposition by sputtering, being the main drawback of this procedure the difficulty of deposition onto recessed, high aspect substrates and the high cost. Electrodeposition is an alternative way to achieve the same objective with lower cost equipment and higher fabrication rates. ${ }^{6}$ Hard template assisted nanowire synthesis based on ordered anodic aluminum oxide (AAO) is widely applied to nanodot, nanowire and nanotube arrays fabrication. ${ }^{6}$ So far, $\mathrm{Ni}, \mathrm{Fe}$ and Co nanowires have been also produced by electrodeposition (ED) into ordered AAO porous substrates evidencing the versatility of this synthesis route.
Although the magnetic properties of the different phases in Sm-Co alloys are well known, very few works have been reported regarding the synthesis of Sm-Co nanowires by electrodeposition (ED), particularly in aqueous medium. ${ }^{7}$ In this aspect, Cojocaru et al. have used a 1 choliride: 2 urea molar eutectic solvent mixture to carried out SmCo electrodeposition in a copper substrate and also in alumina template, ${ }^{14}$ and Chen et al. have deposited SmCo alloy at constant potential, from 1-ethyl-3-methylimidazolium chloride ionic liquid, without template. ${ }^{15}$

In order to improve the morphology and microstructure of these 1D particles, and also the magnetic and/or electronic properties, electrocrystallization conditions must be precisely determined.

In the present work, Sm-Co nanowires were fabricated by ED into AAO templates and further characterized, in order to experimentally determine the value of the reduction potential for the electrodeposition of the alloy in an aqueous medium. The effects of the electrodeposition potential applied during the synthesis process on the composition, crystallography, morphology and on the magnetic properties of the nanostructures are further investigated. This is an original result as no data related to Sm-Co electrodeposited in aqueous medium at these low potentials, and without chelating agents have been reported.

\section{Experimental}

Materials._All reagents, $\mathrm{SmCl}_{3} \cdot 7\left(\mathrm{H}_{2} \mathrm{O}\right)$ (Alfa Aesar), $\mathrm{CoSO}_{4} \cdot 7$ $\left(\mathrm{H}_{2} \mathrm{O}\right.$ ) (Sigma-Aldrich) and $\mathrm{H}_{3} \mathrm{BO}_{3}$ (Cicarelli) were of analytical grade and being used without further purification. Commercial AAO porous templates (about $10^{10}$ pores $\mathrm{cm}^{-2}$ ), with nominal pore diameter of $200 \mathrm{~nm}, 60 \mu \mathrm{m}$ thickness, were purchased by Whatman.

Aqueous solutions were prepared by using $18.2 \mathrm{M} \Omega \cdot \mathrm{cm}^{-1}$ resistance water (Milli-Q, Millipore, Billerica, MA), $\mathrm{pH}$ measurements performed with a combined glass electrode and a digital $\mathrm{pH}$-meter (Altronix). Unless noted, all experiments were performed at room temperature, $(300 \pm 2) \mathrm{K}\left[(27 \pm 2)^{\circ} \mathrm{C}\right]$.

Nanowire/nanotube arrays are obtained by ED; a continuous layer of gold (ca. $30 \mathrm{~nm}$ thick) is thermally sputtered onto one side of the template to serve as an AAO/gold working electrode. ED of $\mathrm{Sm}-\mathrm{Co}$ is carried out in a three-electrode cell, using aqueous solution (ED solution) of $60 \mathrm{mM} \mathrm{CoSO}_{4}+60 \mathrm{mM} \mathrm{SmCl}_{3}$ in $500 \mathrm{mM}$ $\mathrm{H}_{3} \mathrm{BO}_{3}$ as electrolyte, at $\mathrm{pH} 3$ and at room temperature. A high-purity platinum plate is used as a counter electrode and a saturated $\mathrm{Ag} / \mathrm{AgCl}$ 


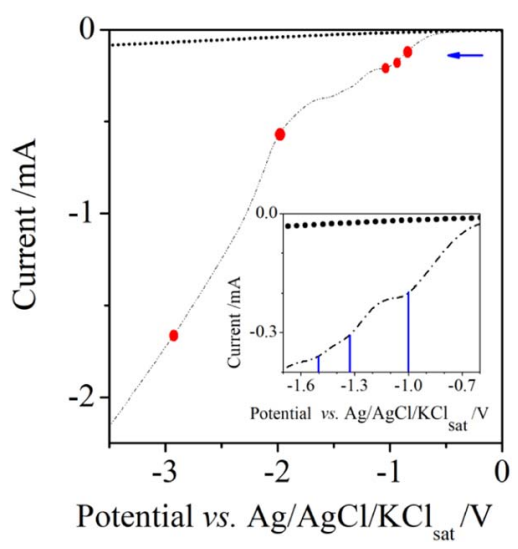

Figure 1. Cyclic voltamperogram in absence (blank solution, dotted line) and in presence of $\mathrm{Sm}^{3+}, \mathrm{Co}^{2+}$ ions (dashed line) at $50 \mathrm{mV} \cdot \mathrm{s}^{-1}$. Working electrode: steel, counter electrode: $\mathrm{Pt}$ and reference electrode: $\mathrm{Ag} / \mathrm{AgCl} / \mathrm{KCl}_{\text {sat }}$. Circles indicate the potential values selected for the electrodeposition. Inset: Magnified vision of the voltamperogram which shows the peaks observed in the reduction of $\mathrm{Sm}^{3+}$ and $\mathrm{Co}^{2+}$.

electrode serving as the reference electrode. Deposition and cyclic voltamperometry studies were performed in an Autolab302N potentiostat/galvanostat device, with the NOVA1.8 measurement software installed.

The resulting microstructures were characterized by scanning (SEM), transmission (TEM) electron microscopy, and X-ray photoelectron spectroscopy (XPS). SEM images of nanowires/nanotubes were recorded on a SEM Carl Zeiss Sigma, and the atomic percentage of $\mathrm{Sm}$ and Co determined by energy-dispersive X-ray spectroscopy (EDS). TEM observations, images and electron diffraction patterns were performed in a Philips CM 200 UT microscope.

XPS experiments were carried out using on a K-Alpha X-ray photoelectron spectroscope (Thermo-Fisher Scientific Co.), with a monochromotized Al-K $\alpha$ X-ray source in vacuum of $10^{-9} \mathrm{mBar}$.

Room temperature magnetic hysteresis loops were measured in a vibrating sample magnetometer (VSM) Lake-Shore 7300, with a maximum field up to $1.5 \mathrm{~T}$, to determine the coercive field $\mu_{0} H_{C}$ and the relative magnetization or squareness $S\left(=M_{R} / M_{S}\right)$.

\section{Results and Discussion}

Mainly two factors are found to affect the deposit composition: one of them is the reduction potential value of the species involved ( $\mathrm{Sm}$, Co) and the other one is the concentration ratio of the components in the feeding solution used for the electrodeposition. The reduction potentials are very different $\left(\mathrm{E}_{\mathrm{Sm} 3+/ \mathrm{Sm}}=-2.304^{17}\right.$ and $\mathrm{E}_{\mathrm{Co} 2+/ \mathrm{Co}}=$ $-0.280^{18}$ ) making difficult to obtain the alloy. An equimolar concentration of Sm:Co is used in the ED solution as indicated by Mishra et al. ${ }^{7}$ These authors conclude that at lower Co concentration (relative to the rare earth), the process rate decreases because there is not enough Co to catalyze the rare earth (RE) deposition.

Brenner ${ }^{19}$ describes the condition where the less noble element is preferentially deposited, and defines this process as anomalous deposition which is a type of abnormal deposition. The induced co-deposition represents another type of abnormal deposition in which an element that cannot be deposited in its pure form, is co-deposited as an alloy. ${ }^{19,20}$

Figure 1 shows the cyclic voltamperograms for the blank solution (dotted line) and for the bath containing $\mathrm{Sm}^{3+}$ and $\mathrm{Co}^{2+}$ ions, measured at $50 \mathrm{mV} \cdot \mathrm{s}^{-1}$ (dashed line). A three electrode electrochemical cell was used with a steel $\left(1 \mathrm{~cm}^{2}\right)$ working electrode, a high-purity platinum plate as a counter electrode and saturated $\mathrm{Ag} / \mathrm{AgCl}$ as the reference electrode. As can be seen, blank solution produces an almost null trace, while for the ED solution three reduction peaks at $-1.0 \mathrm{~V}$, $-1.3 \mathrm{~V}$ and $-1.5 \mathrm{~V}$ can be observed, as is clearly shown in the insert.

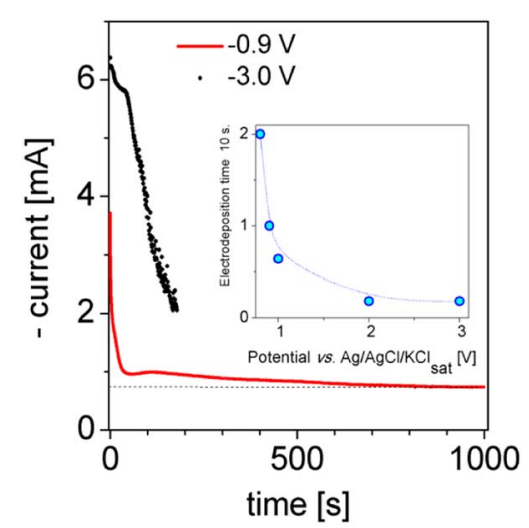

Figure 2. Transient current responses obtained from a solution of $\mathrm{Co}^{2+}$ $60 \mathrm{mM}-\mathrm{Sm}^{3+} 60 \mathrm{mM}$, at applied potentials of $-0.9 \mathrm{~V}$ and $-3.0 \mathrm{~V}$ vs. $\mathrm{Ag} / \mathrm{AgKClsat}$. Inset: Electrodeposition time as a function of the applied potential.

The peak located at $-1.0 \mathrm{~V}$ is related to $\mathrm{Co}^{2+}$ to $\mathrm{Co}_{(\mathrm{s})}$ reduction. The peaks observed at $-1.3 \mathrm{~V}$ and $-1.5 \mathrm{~V}$ arise from the reduction of $\mathrm{Sm}^{3+}$ and $\mathrm{Sm}^{2+}$. There is evidence that the $\mathrm{Sm}-\mathrm{Co}$ deposition follows an induced co-deposition behavior, which was also reported in REtransition metal alloys deposition such as Co with $\mathrm{Tb}^{8}$ or $\mathrm{Gd}^{9}$, where cobalt deposition is inhibited once the rare earth deposition starts. RE electrodeposition at potentials which are more positive than the equilibrium reduction potential is enabled by the catalytic action of cobalt, similar to the induced co-deposition mechanism observed in molybdenum and tungsten. ${ }^{20,22,23}$ Liu et al. observed the deposition of $\mathrm{SmCo}$ and $\mathrm{NdFe}$ at lower potential respect to the reduction potential of $\mathrm{Sm}(\mathrm{III})$ and $\mathrm{Nd}(\mathrm{III})$, which is in the range of $-2.5 \mathrm{~V}$ vs. Saturated Calomel Electrode (SCE). As a consequence, they proposed that a mixed intermediate metal is forms. ${ }^{24}$ That is why the peaks observed at approximately $-1.3 \mathrm{~V}$ and $-1.5 \mathrm{~V}$ are attributed to the reduction of $\mathrm{Sm}^{3+}$ to the intermediate hydroxide species $\mathrm{SmCoOH}\left(\mathrm{Sm}^{2+}\right)$ and the reduction of this intermediate compound to $\mathrm{Sm}_{(\mathrm{s})}$, respectively. This idea is supported by the absence of a buffer solution and the concomitant increment in the local $\mathrm{OH}^{-}$concentration, as a result of the hydrogen electrochemical reaction. It should be noted that although boric acid is used as electrolyte support in the electrodeposition, the $\mathrm{pH}$ of the solution is around 3 , fare away from the $\mathrm{pKa}$ value of boric acid, $\mathrm{pKa}=9.24$, so it is not a buffer solution. ${ }^{24}$ In the present work the solution is not stirred because Panzeri et al. ${ }^{16}$ observed that stirring promotes a high depletion in the Sm content. Additionally, Kolb et al. ${ }^{26}$ attribute the deposition of some metals at more positive potential values to the differences in the work function value $\left(\phi_{M}\right)$ between the metallic electrode and the metallic monolayer deposition. The difference $\phi_{\mathrm{Au}}-\phi_{\mathrm{sm}}=2.4 \mathrm{eV}$ implies a potential shift of $\Delta \mathrm{E}_{\mathrm{red}}=1.2 \mathrm{~V}\left(\Delta \mathrm{E}_{\mathrm{red}}\right.$ $\left.=0.5 \mathrm{~V}(\mathrm{eV})^{-1} \phi_{\mathrm{M}}\right)$ to more positive values, that is, from $\mathrm{E}_{\text {red,Sm }}^{\circ}=$ $-2.3 \mathrm{~V}$ to $-1.1 \mathrm{~V} .^{26,27}$ This shift is explained by the generation of a partial charge in the adatoms, ${ }^{26-28}$ giving a ionic contribution to the chemical bond which reinforces the induced co-deposition effect.

Based on these results five potential values were selected to produce nanostructures by elec-trodeposition. These values $(-0.8 \mathrm{~V},-0.9 \mathrm{~V}$, $-1.0 \mathrm{~V},-2.0 \mathrm{~V}$ and $-3.0 \mathrm{~V}$ ) are marked in Figure 1.

Figure 2 shows typical $J$ vs. $t$ transient curves recorded in the course of the potentiostatic alloy deposition at $-0.9 \mathrm{~V}$ and $-3.0 \mathrm{~V}$. The electrodeposition processes exhibit characteristic stages: first, a maximum of cathodic current corresponding to the initial stage is observed, followed by a stage of non-monotonic current decrease, eventually reaching a constant current stationary regime, which corresponds to the nanostructure growth inside the cylindrical AAO channels. ${ }^{2,8}$

At a potential of $-3.0 \mathrm{~V}$, variations in the current are observed due to the $\mathrm{H}_{2}$ discharge. All electrodeposition experiments were stopped when the total charge value was $800 \mathrm{mC}$; and as a consequence, the total electrodeposition times are different and depend on the applied potential, as illustrated in the inset. 


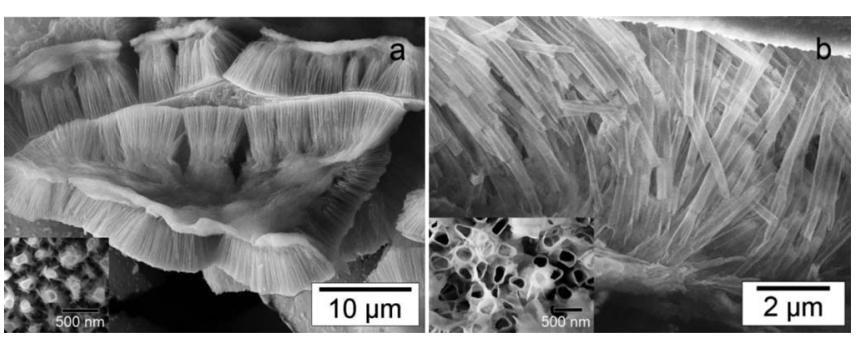

Figure 3. SEM micrographs showing the morphology to the nanostructures obtained at an applied potential of (a) $-0.9 \mathrm{~V}$ (nanowires) and (b) $-3.0 \mathrm{~V}$ (nanotubes).

Morphology and microstructure.-Figure 3 shows SEM micrographs corresponding to the different electrodeposited SmCo arrays, after the partial dissolution of the alumina membrane in $\mathrm{NaOH} 1 \mathrm{M}$ during $60 \mathrm{~min}$. The arrays consist of ordered, parallel and densely distributed nanowires for $-0.9 \mathrm{~V}$ (Figure $3 \mathrm{a}$ ) and nanotubes for $-3 \mathrm{~V}$ (Figure 3b), indicating that pores were optimally filled. Details about the arrays size and morphology are listed in Table I.

Nanowires obtained at $-0.8 \mathrm{~V}$ are short and quite irregular. Nevertheless, the resulting nanowires are long and perfectly ordered for a potential of $-0.9 \mathrm{~V}$, as can be observed in Figure 3a. At this potential Sm-Co nanowires are abundant, uniform and densely packed in a large scale.

At a potential value of $-1.0 \mathrm{~V}$ long, perfectly ordered nanowires are obtained mixed with also regular nanotubes. There are no previous reports on Sm-Co 1D nanostructure obtained at such low potentials. ${ }^{7}$

In this condition, the background of hydrogen discharge associated to high potential values $(-2 \mathrm{~V}$ or $-3 \mathrm{~V})$ can be avoided ${ }^{7}$ without the use of expensive and impractical procedures. ${ }^{14}$

When the potential applied is $-2.0 \mathrm{~V}$ or $-3.0 \mathrm{~V}$ the resulting nanostructures are nanotubes $195 \mathrm{~nm}$ outer diameter and $30 \mathrm{~nm}$ wall thick, leading to an internal diameter of $135 \mathrm{~nm}$ and to a factor $\beta=\left(D_{\text {int }} / \mathrm{D}\right)=$ 0.7 (Figure $3 b$ ). This factor determines the magnetization reversal mechanism in the NT as will be seen below. This NT morphology, appearing for high applied potentials promoting rapid nanostructure growth, probably arises from the strong hydrogen evolution. Fukunaka et al. ${ }^{30}$ and Davis et al. ${ }^{31}$ observed in electrodeposition of transition metal nanotubes, that the hydrogen evolution played a major role in determining wire or tube formation.

The nominal composition, obtained by EDS, is plotted as a function of the applied potential in the electrodeposition process and is shown in Figure 4. At electrodeposition potentials as low as $-0.8 \mathrm{~V}$, $-0.9 \mathrm{~V}$ and $-1.0 \mathrm{~V}$, the nanostructure nominal composition approximately corresponds to Sm 10 at.\% and Co 90 at.\%, in agreement with that of the $\mathrm{Sm}_{2} \mathrm{Co}_{17}$ equilibrium phase in this binary system. On the other hand, at potentials usually applied for rare earth deposition such as $-2.0 \mathrm{~V}$ and $-3.0 \mathrm{~V}$, the nominal composition changes to the equiatomic one, $\mathrm{Sm} 50$ at.\% and Co 50 at.\%.

Table I. Morphological parameters corresponding to the electrodepositions performed at different potentials. The cylinder length $\mathrm{L}$, the internal structure (nanowires NWs or nanotubes NTs), their external diameter $D$, the NTs wall thickness, the respective aspect ratios $a_{R}(=\mathrm{L} / \mathrm{D})$ and $\left.\beta\left(=\left(D-2 t_{W}\right) / D\right)\right)$ factors are listed.

\begin{tabular}{ccccccc}
$-E[\mathrm{~V}]$ & $L[\mu \mathrm{m}]$ & Morphology & $D[\mathrm{~nm}]$ & $t_{W}[\mathrm{~nm}]$ & $a_{R}$ & $\beta$ \\
\hline 0.8 & $1.0 \pm 0.5$ & NW & $178 \pm 10$ & - & 5.5 & - \\
0.9 & $6 \pm 1$ & NW & $180 \pm 10$ & - & 33 & - \\
1.0 & $5 \pm 1$ & NW/NT & $160 \pm 10$ & $35 \pm 3$ & 33 & - \\
2.0 & $20 \pm 3$ & NT & $190 \pm 10$ & $30 \pm 3$ & 100 & 0.7 \\
3.0 & $18 \pm 3$ & NT & $205 \pm 10$ & $31 \pm 3$ & 100 & 0.7
\end{tabular}

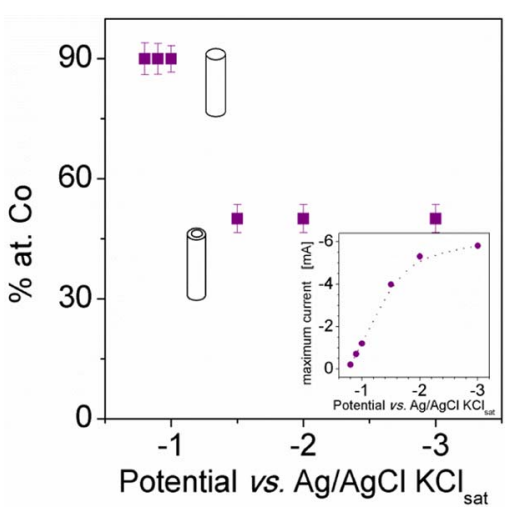

Figure 4. EDS results of atomic percent of Co expressed as a function of applied potential.

There is some evidence that the deposition rate of Sm in Sm-Co alloys considerably increases when the potential applied is near to $-2.0 \mathrm{~V}$. In this sense, our results are consistent with previously published data for electrodeposition of Sm-Co films from aqueous media. ${ }^{7}$

In order to determine the degree of crystallinity and the phases in the nanostructures, XRD experiments were performed for all the samples. Diffractograms (not shown) were practically featureless, with only a few small peaks arising from the alumina template or the gold layer. Then, crystallographic properties were further explored by TEM.

Figure 5 shows bright field images of typical nanostructures resulting for a potential value of $-0.9 \mathrm{~V}$ - Figure $5 \mathrm{a}-$ and $-3 \mathrm{~V}-$ Figure $5 \mathrm{~b}$. A subtle film is frequently found around the nanostructures, which contains $\mathrm{CoO}$, most probably formed during the sample
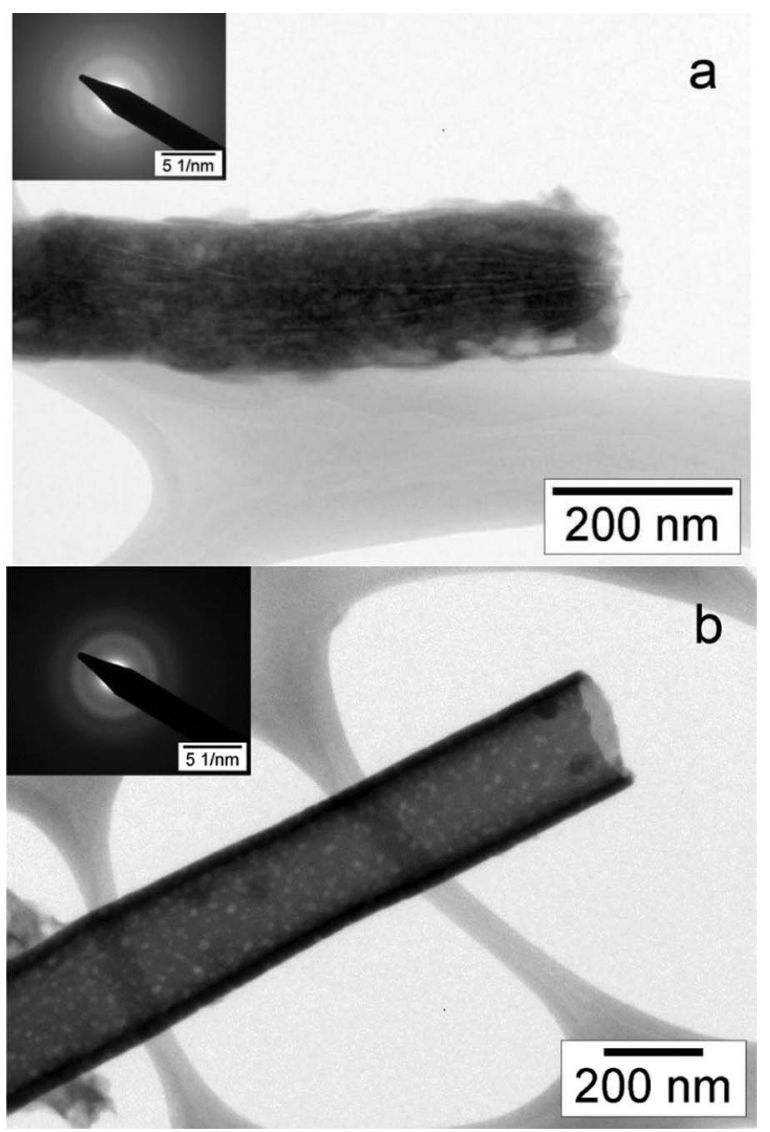

Figure 5. TEM images of Sm-Co nanostructures obtained at a potential of (a) $-0.9 \mathrm{~V}$ (nanowires) and (b) $-3.0 \mathrm{~V}$ (nanotubes). 


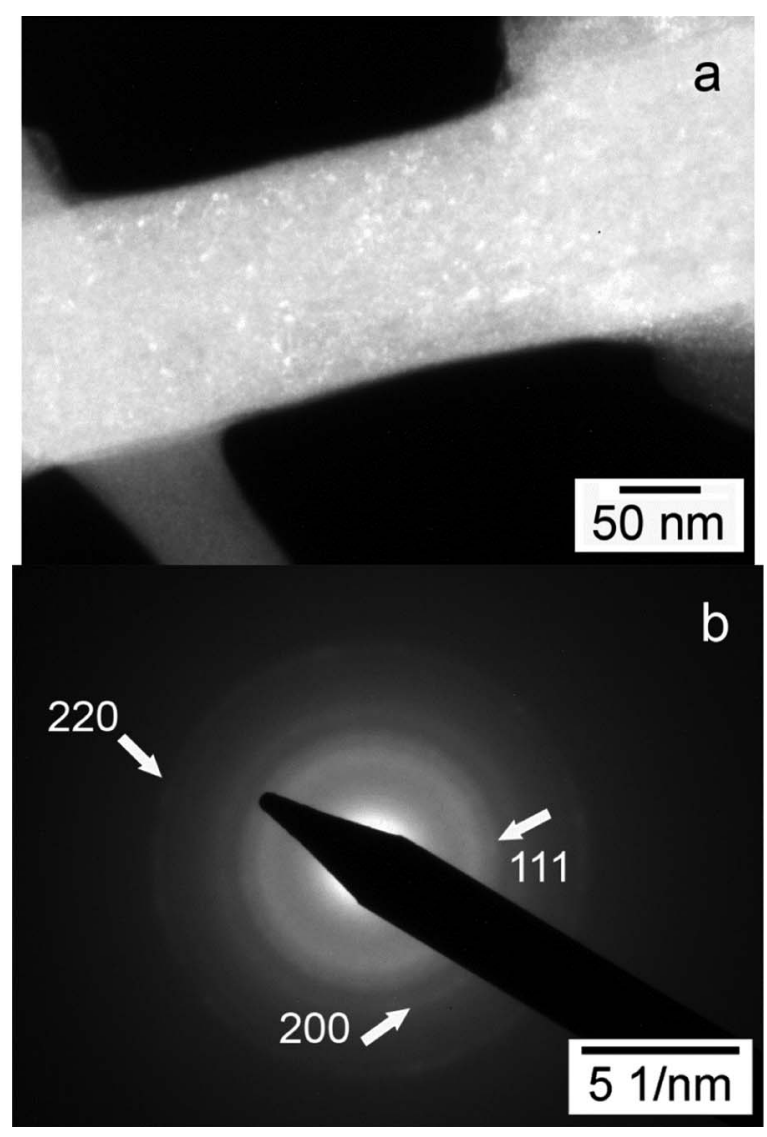

Figure 6. (a) Dark field image of a NT obtained at $-3 \mathrm{~V}$ taken with a spot belonging to the crystalline nanophase $(\sim 5 \mathrm{~nm})$ indicating that these small crystallites are $\mathrm{CoO}$ formed onto the NTs and NWs surfaces. (b) Electron diffraction rings corresponding to the $\mathrm{CoO}$ phase (arrows).

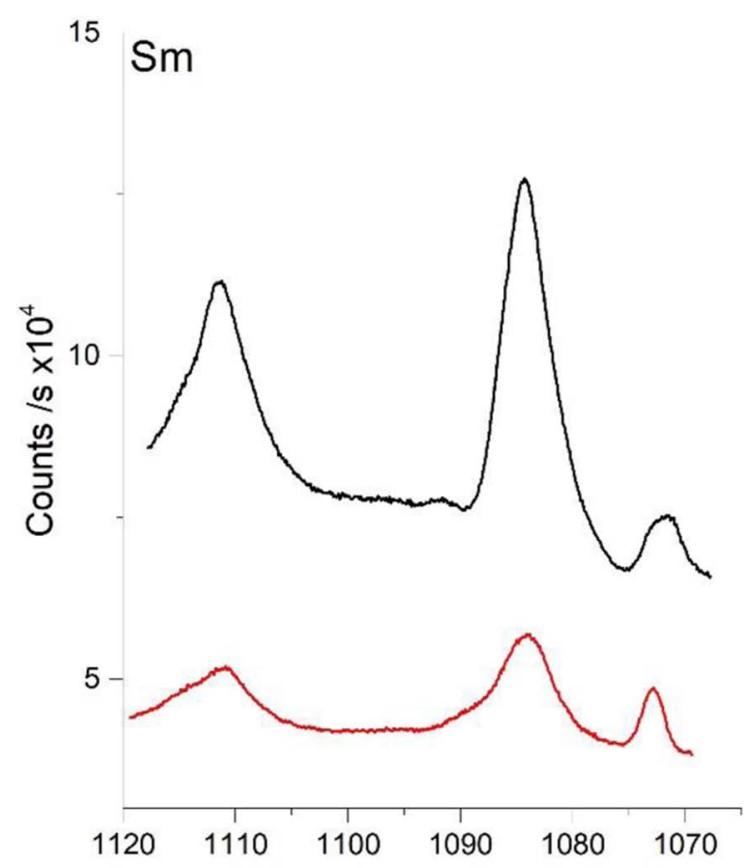

preparation for TEM. The selected area diffraction pattern in the inset of Figure 5a indicates that NWs are amorphous. The diffraction pattern of nanotubes obtained at $-3 \mathrm{~V}$, Figure $5 \mathrm{~b}$, also indicates an amorphous phase and small crystals of about $5 \mathrm{~nm}$.

The dark field image taken with a spot belonging to this crystalline nanophase is shown in Figure 6, confirming that these small crystallites are $\mathrm{CoO}$ formed onto the NTs and NWs surfaces. Then, samples of both morphologies are nominally amorphous, in agreement with our $\mathrm{X}$-ray diffraction results commented above.

Figure 7 shows XPS experiments for nanowires in the region of Sm, Figure $7 \mathrm{a}$, and Co signals, Figure $7 \mathrm{~b}$, before and after stripping the surface of the nanowires with an Ar beam. Sm peaks before stripping, which correspond to $3 \mathrm{~d}_{3 / 2}$ and $3 \mathrm{~d}_{5 / 2}$, appear at $1084.3 \mathrm{eV}$ and $1111.5 \mathrm{eV}$ $(\Delta \mathrm{E}=27.2 \mathrm{eV})$, respectively, and at $1084.0 \mathrm{eV}$ and $1110.8 \mathrm{eV}(\Delta \mathrm{E}=$ $26.8 \mathrm{eV}$ ) after that procedure. The slight change and the decrease in the peaks separation, are related to the fact that the metallic character of the surface increases as a result of the stripping. ${ }^{32-34}$

These energy values are slightly higher than expected for metallic $\mathrm{Sm}$, because in the SmCo alloy, Sm is attached to Co, which is more electronegative. In addition, as shown in Figure 7b, the peaks corresponding to cobalt oxide were observed, and with the first pickling, the Co metallic signal increases considerably. ${ }^{35,36}$ It should be noted that as the AAO template is dissolved with $\mathrm{NaOH} 1 \mathrm{M}$ in the present protocol, then, the metallic oxides could be formed at this stage.

Magnetic hysteresis properties.-The effect of the different potentials applied during electrodeposition on the magnetic properties was investigated by measuring the room temperature hysteresis loops. Low absolute potential values lead to higher Co content in the amorphous phase and also to NW morphology, both features promoting higher coercivity values. Figure 8 shows typical hysteresis loops of ordered Sm-Co nanowires, measured inside the AAO templates, for two extreme applied potentials as $0.9 \mathrm{~V}$ (a) and $-3.0 \mathrm{~V}$ (b). The hysteresis loops are measured with the external field applied parallel (PA) and perpendicular (PE) to the major axis of nanowires/nanotubes.

Considering that NWs and NTs are amorphous quite low crystalline anisotropy may be expected and only shape anisotropy is considered. Shape anisotropy and the corresponding shape anisotropy field in NWs

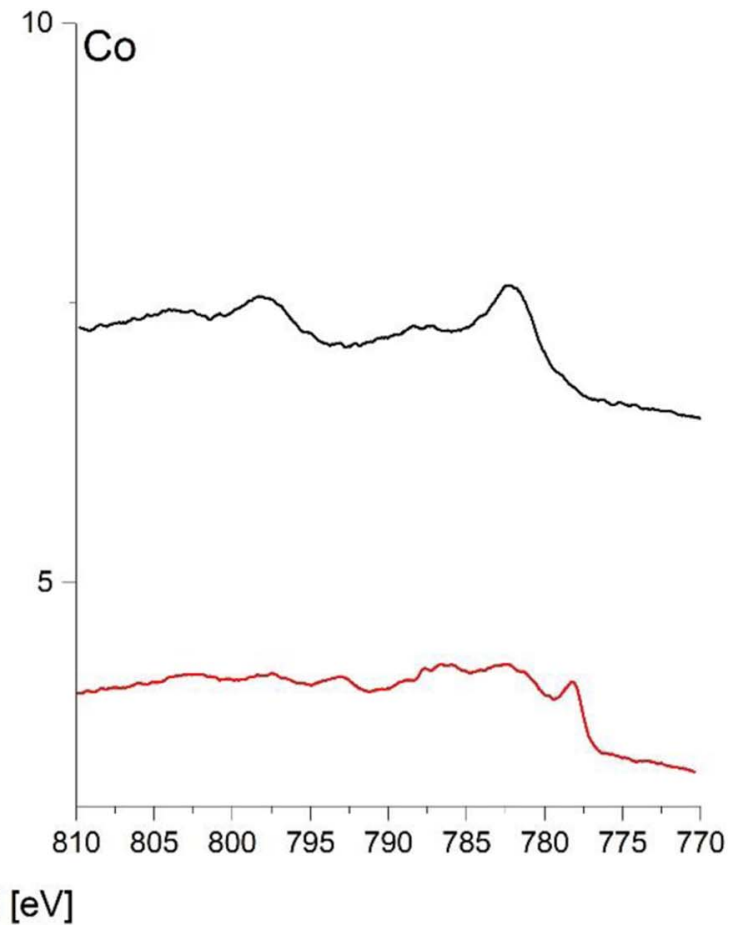

Figure 7. XPS experiments for nanowires in the region of Sm (a) and Co signals (b), before (black) and after (red) stripping with Ar. 

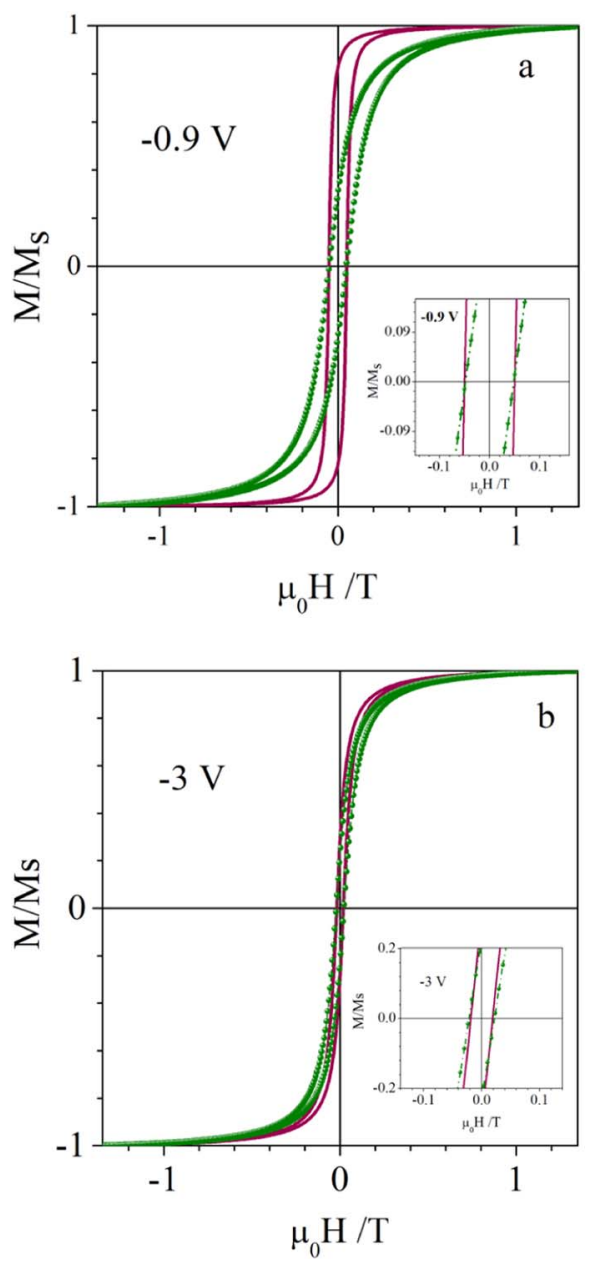

Figure 8. Hysteresis loops of Sm-Co electrodeposited at (a) $0.9 \mathrm{~V}$ (NWs) and at (b) $-3.0 \mathrm{~V}$ (NTs) measured with the external field applied parallel (PA) and perpendicular $(\mathrm{PE})$ to the long nanowire axis.

are estimated as:

$$
\begin{gathered}
K_{s h}^{N W}=\frac{1}{2} \Delta N \mu_{0} M_{S}^{2} \\
\mu_{0} H_{s h}(T)=\frac{1}{2} \Delta N \mu_{0} M_{S}(T)
\end{gathered}
$$

with $\Delta N$ the difference between of demagnetizing factor along the major wire axis and that in a normal direction, and $\mu_{0} M_{S}$ the NWs' saturation polarization. For NWs with nominal composition close to the $\mathrm{Sm}_{2} \mathrm{Co}_{17}$ phase and an aspect ratio of 33 , the saturation polarization may be approximated by the value corresponding to this phase $\mu_{0} M_{S}^{N W}=1.25 \mathrm{~T}(298 \mathrm{~K})$. The desmagnetizing factor of finite nanowires along the major length may be estimated as proposed by Landeros et al., ${ }^{37}$ leading to $N_{z}=0.044$. Then, $\Delta N^{N W}=0.43$, $K_{s h}^{N W}=2.67 \times 10^{5} \mathrm{~J} \mathrm{~m}^{-3}$ and $\mu_{0} H_{s h}^{N W}(T)=0.268 T$.

For nanotubes obtained at $-2.0 \mathrm{~V}$ and $-3.0 \mathrm{~V}$ the demagnetizing factor along the major axis is negligible $\left(\Delta N^{N T}=0.5\right)$ and the shape anisotropy constant and field are respectively $K_{s h}^{N T}=0.95 \times 10^{5} \mathrm{~J} \mathrm{~m}^{-3}$ and $\mu_{0} H_{s h}^{N T}(T)=0.17 T$.

These magnitudes indicate that both nanostructures are quite soft, as expected for amorphous samples, and that larger coercive fields and magnetostatic shape effects are expected in nanowires than in nanotubes, as observed in Figure 8. In both types of nanostructure the effective easy axis is parallel to the long axis.

Figure 9 summarizes coercivity $\left(\mu_{0} \mathrm{Hc}\right)$ and squareness $(\mathrm{S}=$ $\mathrm{M}_{\mathrm{R}} / \mathrm{M}_{\mathrm{S}}$ ) for SmCo nanotube and nanowires arrays, as functions of the electrodeposition potential. As observed in Figure 8 for the ex-

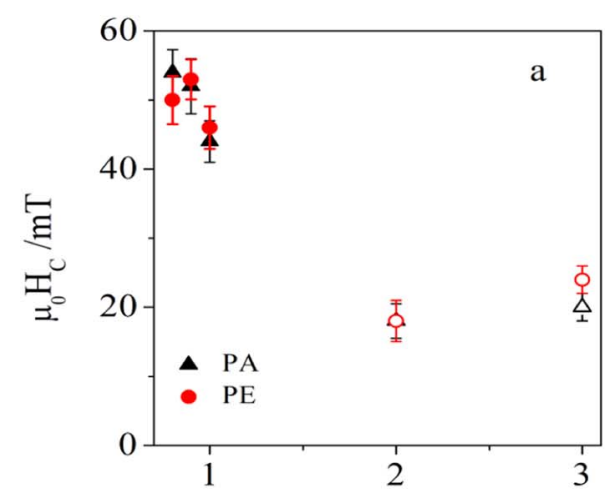

Potential vs. $\mathrm{Ag} / \mathrm{AgCl} \mathrm{KCl}{ }_{\text {sat }} / \mathrm{V}$

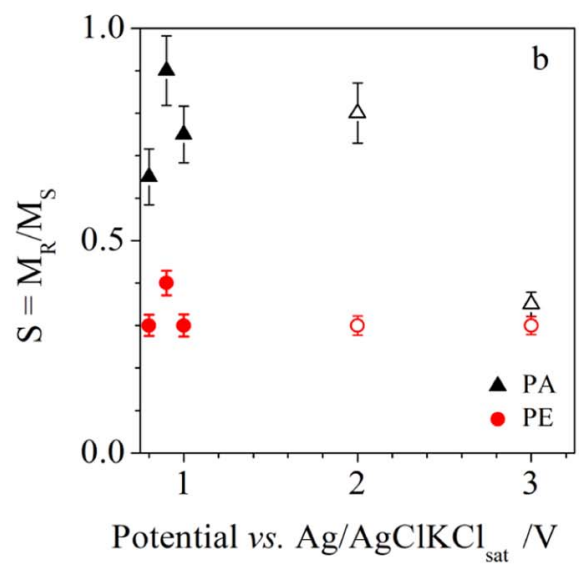

Figure 9. (a) Coercive field $\mu_{0} \mathrm{Hc}$ and (b) squareness $\mathrm{S}$ as functions of the electrodeposition potential. Full symbols correspond to NWs and open symbols to NTs.

treme potentials values, no significant difference between the coercivity in configurations PA and PE is observed in all the cases. Coercivity decreases as the potential applied in the electrodeposition increases due to the transition from NW to NT morphology and the decrease in the Co content in the nanostructure. Squareness (S) also decreases for higher potential while the difference between the two orientations becomes smaller as discussed in Figure 8.

The magnetization reversal mechanisms operating in these amorphous 1D nanostructures, with NW and NT morphologies and also different compositions, may be characterized by measuring the coercive field and the relative remanence (S) as functions of the angle $\theta$ between the long axis of NWs and NTs, and the applied magnetic field. The results for samples synthesized at different electrodeposition potentials are shown in Figure 10. It may be observed that, despite the different nanostructures' morphology and composition, the shape of curves in Figure 10a $\mu_{0} \mathrm{H}_{\mathrm{C}}(\theta)$-are quite similar, and the same is found for curves $S(\theta)$ shown in Figure $10 \mathrm{~b}$.

Coercivity angular variation shown in Figure 10a is similar to curves reported by other authors in NWs ${ }^{38-40}$ and NTs. ${ }^{41-43}$ Curves in Figure 10b, displaying the monotonic decrease of the relative remanence with the angle $\theta$, are also similar to those reported by these latter authors.

The non-monotonic behavior of curves $\mu_{0} H_{C}(\theta)$ arises from the transition from an initial reversal regime to another one, taking place at a given critical angle depending on the sample geometry and magnetic hardness. The initial reversal mechanism involves the localized nucleation by curling, and further expansion of vortex-like domain walls. At higher angles, where the applied field is almost perpendicular to the NW/NT long axis, the mechanism changes to one involving nucleation by localized coherent rotation and further expansion of transverse Bloch-like walls. This transition is observed in NWs 

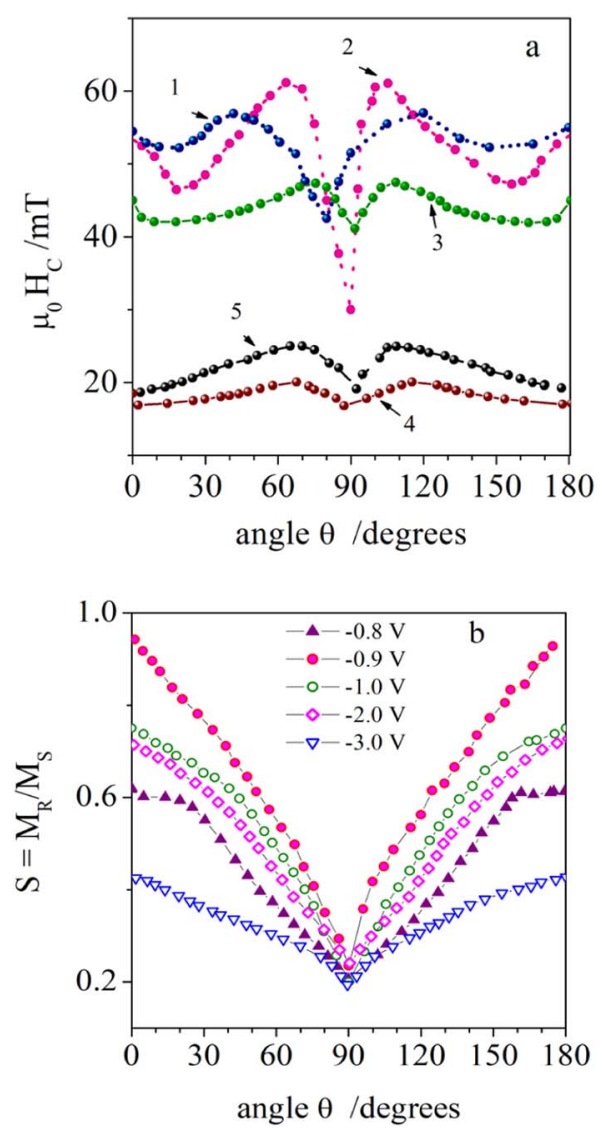

Figure 10. Angular dependence of (a) the coercive field and (b) the relative remanence, corresponding to the NWs and NTs electrodeposited. $\theta$ is the angle between the major axis of the nanostructure (easy axis direction) and the applied magnetic field.

and NTs at somewhat different critical angles $\theta_{C}^{N W} \approx 45-65^{\circ}$ and $\theta_{C}^{N T} \approx 70-75^{\circ}$. In the case of magnetically soft NTs, this critical angle increases with the outer diameter, being about $55^{\circ}$ for a diameter of $\mathrm{d}=50 \mathrm{~nm}$ while for $\mathrm{d}=65 \mathrm{~nm}$, the critical transition angle reaches $\theta_{C}^{N T}=67^{\circ}$. One should note that the outer and inner diameters of a NT array will highly influence the type of DW that is energetically more favorable to nucleate. In addition to the transition in the reversal mechanism, another factor promoting this type of non-monotonic coercivity curves is the dipolar interactions between nanocylinders, as reported by Bender et al., ${ }^{44}$ especially in our case of large diameter 1D nanostructure.

\section{Conclusions}

This work demonstrates for the first time that it is possible the synthesize Sm-Co 1D nanostructures by electrodeposition of the alloy in aqueous medium, at quite low potential values and without chelating agents.

For the first time long, uniform and densely packed Sm-Co nanowire arrays were prepared by electrodeposition inside the cylindrical pores of AAO templates, at quite low electrodeposition potentials such as $-0.8 \mathrm{~V},-0.9 \mathrm{~V}$ and $-1.0 \mathrm{~V}$. Samples were amorphous NWs, with a nominal composition close to that of the equilibrium stoichiometric phase $\mathrm{Sm}_{2} \mathrm{Co}_{17}$. At very high potential as $-3.0 \mathrm{~V}$, long and regular nanotubes $190 \mathrm{~nm}$ in outer diameter and of $30 \mathrm{~nm}$ thick walls form with the equiatomic SmCo composition, mainly due to the strong hydrogen evolution.

NWs and NTs are soft ferromagnetic, with the easy axis parallel to the sample major axis and magnetic hardness is governed by the shape anisotropy through the saturation polarization (Co content) with little influence of geometry (aspect ratio).

The angular variation of the coercive field with the angle $\theta$ between the sample major axis and the applied field is consistent with a transition from an initial reversal mechanism, involving the localized nucleation by curling, and further expansion of vortex-like domain walls to another one. In fact, at higher angles, where the applied field is almost perpendicular to the NW/NT long axis, the mechanism changes to one involving nucleation by localized coherent rotation and further expansion of transverse Bloch-like walls.

\section{Acknowledgments}

The authors thank SeCyT-UNC, FonCyT and CONICET Argentina for the financial support given to this work.

\section{ORCID}

Silvia E. Urreta (D) https://orcid.org/0000-0003-4502-1182

\section{References}

1. T. Sun, Y. S. Zhang, B. Pang, D. C. Hyun, M. Yang, and Y. Xia, Angew. Chemie Int. Ed., 53, 2 (2014).

2. G. R. S. Andrade, C. C. Nascimento, Z. M. Lima, E. Teixeira-Neto, L. P. Costa, and I. F. Gimenez, Appl. Surf. Sci., 399, 573 (2017).

3. L. G. Astafyeva, V. K. Pustovalov, and W. Fritzsche, Photonics Nanostructures Fundam. Appl., 26, 35 (2017)

4. M. Yu, Y. Ma, J. Liu, X. Li, S. Li, and S. Liu, Appl. Surf. Sci., 390, 266 (2016).

5. J. K. Carrow and A. K. Gaharwar, Macromol. Chem. Phys., 216, 248 (2015).

6. J. S. Riva, G. Pozo-López, A. M. Condó, J. M. Levingston, L. M. Fabietti, and S. E. Urreta, J. Alloys Compd., 709, 531 (2017).

7. R. Mishra and E. J. Podlaha, ECS Transactions, First International Symposium on Electrodeposition of Nanoengineered Materials, Meeting of The Electrochemical Society, Denver, CO, (2005).

8. R. Mishra and E. J. Podlaha, J. Electrochem. Soc., 153(6), C422 (2006).

9. R. Mishra and E. J. Podlaha, Electrochem. Solid State Lett., 9(12), C199 (2006).

10. V. Sharma, S. Kumari, and B. K. Kuanr, AIP Adv., 8, 56232 (2018).

11. H. Hegde, S. U. Jen, K. Chen, and F. J. Cadieu, J. Appl. Phys., 73, 5926 (1993).

12. W. Xiang, L. Zuo-Yi, L. Zhen, C. Chang-Bo, H. Zhi-Xin, L. Hong-Wei et al., Chinese Phys. Lett., 20, 281 (2003).

13. Q. Wang, L. Zheng, S. An, T. Zhang, and C. Jiang, J. Magn. Magn. Mater, 331, 245 (2013).

14. P. Cojocaru, L. Magagnin, E. Gomez, and E. Vallés, Mat. Lett., 65, 3597 (2011).

15. Y. Chen, H. Wang, and B. Li, RSC Adv., 5, 39620 (2015).

16. G. Panzeri and L. Magagnin, ECS Trans., 75, 31 (2016).

17. E. David and R. Lide, CRC Handbook of Chemistry and Physics, CRC Press. Taylor Fr. Boca Rat. FL. (2005).

18. P. Vanysek, "Electrochemical series", in Handbook of chemistry and physics, Hand B., 1 (2000).

19. A. Brenner, Electrodeposition of Alloys, Vol I \& II, New York, (1963).

20. D. Landolt, Plat. Surf. Finish., 88, 70 (2001).

21. E. Gómez, P. Cojocaru, L. Magagnin, and E. Valles, J. Electroanal. Chem., 658, 81 (2011).

22. N. Zech, J. Electrochem. Soc., 146, 2892 (1999).

23. Y. Zhuang and E. J. Podlaha, J. Electrochem. Soc., 150, C225 (2003).

24. P. Liu, Y. P. Du, Q. Q. Yang, Y. X. Tong, and G. A. Hope, J. Electrochem. Soc., 153 C57 (2006).

25. G. G. Manov, N. J. DeLollis, and S. F. Acree, J. Res. Nat. Bureau Standards, 33, 287 (1944).

26. D. M. Kolb, M. Przasnyski, and H. Gerischer, J. Electroanal. Chem., 54, 25 (1974).

27. C. Sánchez and E. Leiva, J. Electroanal. Chem., 458, 183 (1998).

28. V. Sudha and M. V. Sangaranarayanan, J. Chem. Sci., 117, 207 (2005).

29. K. S. Napolskiia, I. V. Roslyakova, A. A. Eliseeva, D. I. Petukhova, A. V. Lukashina, S. F. Chenb, C. P. Liub, and G. A. Tsirlina, Electrochim. Acta, 56, 2378 (2011).

30. Y. Fukunaka, M. Motoyama, Y. Konishi, and R. Ishii, Electrochem. Solid-State Lett., 9, 62 (2006)

31. D. M. Davis and E. J. Podlaha, Electrochem. Solid-State Lett., 8, D1 (2005).

32. A. Reisner, D. Kasinathan, S. Wirth, L. H. Tjeng, and S. G. Altendorf,, 117 EPL, 47001, 1 (2017).

33. Y. Wei, H. Fan, and R. Wang, App. Surf. Sci., 459, 63 (2018).

34. Y. H. Liu, Y. D. Yan, M. L. Zhang, D. B. Ji, Peng Li, T. Q. Yin, Pu Wang, Y. Xue, X. Y. Jing, W. Han, M. Qiu, and D. Hu, J. All. Comp., 772, 978 (2019).

35. C. C. Naik and A. V. Salker, Mater. Res. Express, 6, 066112 (2019).

36. https://xpssimplified.com/elements/cobalt.php.

37. P. Landeros, J. Escrig, D. Altbir, D. Laroze, J. d'Albuquerque, E. Castro, and P. Vargas, Phys. Rev. B, 71, 0944351 (2005)

38. G. C. Han, B. Y. Zong, and P. Luo, J. of Appl. Phys., 93 (11), 9202 (2003). 
39. R. Lavin, J. C. Denardin, A. P. Espejo, A. Cortés, and H. Gómez, J. Appl. Phys., 107, $09 B 504$ (2010).

40. R. Lavín, J. C. Denardin, J. Escrig_ D. Altbir, A. Cortés, and H. Gómez, J. Appl. Phys., 106, 103903 (2009).

41. O. Albrecht, R. Zierold, S. Allende, and J. Escrig, J. Appl. Phys., 109, 093910 (2011)
42. M. P. Proenca, C. T. Sousa, J. Escrig, J. Ventura, M. Vazquez, and J. P. Araujo, J. Appl. Phys., 113, 093907 (2013).

43. M. P. Proenca, C. T. Sousa, J. Ventura, J. P. Araujo, J. Escrig, and M. Vazquez, Spin, 2 (4), 1250014 (2012).

44. P. Bender, F. Krämer, A. Tschöpe, and R Birringer, J. Phys. D: Appl. Phys., 48, 145003 (2015). 